\title{
A Novel Vital-Sign Sensor Based on a Self-Injection-Locked Oscillator
}

\author{
Fu-Kang Wang, Student Member, IEEE, Chien-Jung Li, Member, IEEE, Chieh-Hsun Hsiao, Student Member, IEEE, \\ Tzyy-Sheng Horng, Senior Member, IEEE, Jenshan Lin, Fellow, IEEE, Kang-Chun Peng, Member, IEEE, \\ Je-Kuan Jau, Jian-Yu Li, Student Member, IEEE, and Cheng-Chung Chen
}

\begin{abstract}
A novel vital-sign sensor with a self-injection-locked oscillator and a frequency demodulator to reduce system complexity and improve sensitivity is proposed. The theory provides a delta-sigma model to account for the excellent signal-to-noise spectral density ratio in a parametric study of the sensitivity performance. Then, the experiments verify the sensing principle and the predicted performance. Accordingly, a prototype sensor with high sensitivity is realized for noncontact cardiopulmonary monitoring, achieving a long sensing distance without the need for a low-noise amplifier. The sensing distance can grow four times longer by doubling the operating frequency. Furthermore, the sensor using a swept frequency can eliminate the null detection points and the external radio interference. As an experimental result, the sensor, which is placed $4 \mathrm{~m}$ away from the subject, can reliably detect the heartbeat signal an the operating frequency of 3.6 GHz and an output power level of $0 \mathrm{dBm}$.
\end{abstract}

Index Terms-Delta-sigma model, frequency demodulator, selfinjection-locked oscillator, vital-sign sensor.

\section{INTRODUCTION}

D UE TO the convenience of wireless networks, wireless healthcare service, which provides wireless data access to home-monitoring healthcare devices, has become prevalent in many countries [1]. With this service, a health-monitoring system can put patients at home under surveillance for recognizing health problems and deliver real-time vital-sign information to a remote caregiver using the current wireless infrastructure. Recently, Doppler radars have been widely used for noncontact cardiopulmonary monitoring [2]-[15]. The advantages of the specific monitoring include reduction in the patients' discomfort during the application of the monitoring process and the potential for widespread adoption. Based on

Manuscript received July 03, 2010; revised October 06, 2010; accepted October 06, 2010. Date of publication November 11, 2010; date of current version December 10, 2010. This work was supported in part by the National Science Council, Taiwan, under Grant 97-2221-E-110-034-MY3, Grant 97-2221-E-110-035-MY3, Grant 97-2628-E-110-041-MY3, and Grant 99-2622-E-110-003-CC1 and by the Department of Industrial Technology, Taiwan, under Grant 98-EC-17-A-01-S1-104. This paper is an expanded paper from the IEEE MTT-S International Microwave Symposium, May 23-28, 2010, Anaheim, CA

F.-K. Wang, C.-J. Li, C.-H. Hsiao, and T.-S. Horng are with the Department of Electrical Engineering, National Sun Yat-Sen University, Kaohsiung 804, Taiwan (e-mail: d983010020@student.nsysu.edu.tw; jason@ee.nsysu.edu.tw).

J. Lin is with the Department of Electrical and Computer Engineering, University of Florida, Gainesville, FL 32611 USA. (e-mail: jenshan@ufl.edu).

K.-C. Peng is with the Department of Computer and Communication Engineering, National Kaohsiung First University of Science and Technology, Kaohsiung 811, Taiwan (e-mail: peterpkg@ccms.nkfust.edu.tw).

J.-K. Jau, J.-Y. Li, and C.-C. Chen are with the Information and Communication Research Laboratories, ITRI, Hsinchu 310, Taiwan (e-mail: jekuan@itri. org.tw).

Digital Object Identifier 10.1109/TMTT.2010.2087349



Fig. 1. Conventional vital-sign sensor architecture.

the Doppler radar technology, Fig. 1 illustrates the most general architecture of the line-of-sight short-range sensor for detecting vital-sign signals [5]-[15]. An RF continuous wave (CW) signal is generated by the voltage-controlled oscillator (VCO) and emitted by the transmit antenna toward the subject. The time-varying chest wall position caused by cardiopulmonary phenomena phase modulates the transmitted signal. The sensor then receives the signal reflected back from the subject via the receive antenna and demodulates it to yield the vital-sign information. Since the phase-noise reduction caused by range correlation was experimentally evaluated in [8], the direct-conversion architectures with free-running oscillators are able to detect low-frequency cardiopulmonary signals without using the phase-locked loops. The null detection points encountered every quarter wavelength from the sensor to the subject can be avoided with the help of a quadrature architecture [8]-[10]. However, the quadrature imbalance often introduces residual phase error to degrade the detection accuracy [11]. The two-tone transmission system in [12] also can resolve the null detection point problem, but it is at the cost of larger bandwidth usage. Since the sensor with higher operating frequency is more sensitive to small chest wall motion, it has been seen that the harmonic sensing architecture [13] and the millimeter-wave circuits [14], [15] are developed for this purpose. However, the design for these sensors is still challenging with respect to robustness, power consumption, and cost. Moreover, most of the vital-sign sensors reported to date operates at a fixed frequency and lack effective mechanism to prevent cofrequency interference.

In this study, a noncontact vital-sign sensor using a VCO with an injection port and a frequency demodulator is proposed for the first time. As shown in Fig. 2, the architecture transmits and receives the signal via the VCO's output and injection port, respectively, to bring the VCO into a self-injection-locking state. The frequency demodulator then extracts the vital-sign signals from the VCO output. The combination of the self-injection-locking and frequency demodulation processes yields a delta-sigma noise-shaping mechanism to achieve a high 


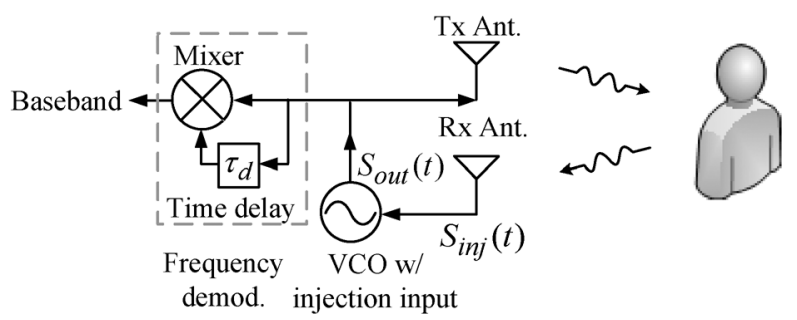

Fig. 2. Proposed vital-sign sensor architecture.

signal-to-noise spectral density ratio (SNR) in the demodulator output. On the basis of this mechanism, the sensor inherently has high sensitivity without using a low-noise amplifier at the front-end. Although the proposed architecture involves a novel sensing principle, the null detection points still exist when the received injection signal into the VCO is quadrature phase with the VCO output signal. For improvement, the architecture allows the VCO to sweep its output frequency, which consequently not only resolve the null detection point problem but also protect the sensor from external radio interference. The authors have covered the architecture and interference aspects and provided brief theory and examples in the preliminary publication of this research [16]. This paper substantially expands [16] to include a delta-sigma model with experimental validation for a parametric study of the SNR gain performance. In addition, this paper further discusses the elimination of the null detection points and demonstrates the vital-sign sensing capability at different frequencies and distances.

\section{Delta-Sigma Model}

Fig. 3(a) displays the block diagram of an oscillator under injection. The oscillator is modeled with an amplifier and a feedback path. The voltage gain of the amplifier is included in the feedback-path impulse response. The feedback path forms a loop to establish the oscillation condition. It should be noted that the feedback path includes a tank circuit and a summing point to allow the injection of signals. The tank circuit has a resonant frequency to determine the frequency of a free-running oscillator. Fig. 3(b) illustrates the vector representation of signals shown in Fig. 3(a) [17]. In Fig. 3(b), $S_{\text {osc }}(t)$ represents the inherent oscillation signal with instantaneous frequency $\omega_{\text {osc }}(t)$ and constant amplitude $E_{\mathrm{osc}}, S_{\mathrm{inj}}(t)$ represents the injection signal with instantaneous frequency $\omega_{\text {inj }}(t)$ and constant amplitude $E_{\text {inj }}$, and $S_{\text {out }}(t)$ is the resultant oscillator output signal under injection with instantaneous frequency $\omega_{\text {out }}(t)$ and constant amplitude $E_{\text {out. }}$.

Based on Adler's analysis [17], $S_{\text {out }}(t)$ can be regarded as a vector rotating clockwise with a beat frequency $d \alpha(t) / d t$ with respect to $S_{\mathrm{inj}}(t)$, and $\alpha(t)$ is the phase difference from $S_{\mathrm{inj}}(t)$ to $S_{\text {osc }}(t)$. Thus, the instantaneous VCO output frequency $\omega_{\text {out }}(t)$ is represented as

$$
\omega_{\text {out }}(t)=\omega_{\text {inj }}(t)+\frac{d \alpha(t)}{d t}
$$

where

$$
\frac{d \alpha(t)}{d t} \approx-\frac{\omega_{\mathrm{osc}}(t)}{2 Q_{\mathrm{tank}}} \frac{E_{\mathrm{inj}}}{E_{\mathrm{osc}}} \sin \alpha(t)+\Delta \omega_{\mathrm{osc}}(t)
$$

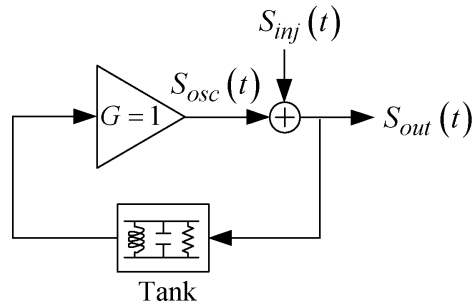

(a)

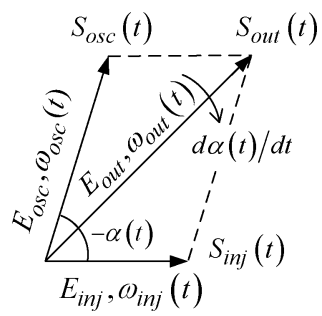

(b)
Fig. 3. Illustration of the injection-locking mechanism. (a) Block diagram of an oscillator under injection. (b) Vector diagram of the signals shown in (a).

is the well-known Adler's equation [17] under the assumption of weak injection level (i.e., $E_{\text {inj }} / E_{\text {osc }} \ll 1$ ). In (2), $Q_{\text {tank }}$ is a quality factor of the VCO's tank circuit, and

$$
\Delta \omega_{\mathrm{osc}}(t)=\omega_{\mathrm{osc}}(t)-\omega_{\mathrm{inj}}(t)
$$

denotes the instantaneous frequency separation between the oscillation and injection signals.

When the VCO enters the self-injection-locking state by inserting the output signal into the injection port via a certain path, all of the signals in Fig. 3(b) can be further treated to have the same frequency $\omega_{\text {osc }}$ with a different instantaneous phase variation, and their instantaneous frequencies are then written as

$$
\begin{aligned}
& \omega_{\mathrm{osc}}(t)=\omega_{\mathrm{osc}}+\frac{d \phi_{n, \mathrm{osc}}(t)}{d t} \\
& \omega_{\mathrm{inj}}(t)=\omega_{\mathrm{osc}}+\frac{d \phi_{n, \text { inj }}(t)}{d t} \\
& \omega_{\text {out }}(t)=\omega_{\mathrm{osc}}+\frac{d \phi_{n, \mathrm{out}}(t)}{d t} .
\end{aligned}
$$

Note that, in (4)-(6), $\phi_{n, \text { inj }}(t)$ and $\phi_{n, \text { out }}(t)$ are mutually dependent, and both are dependent on $\phi_{n, \text { osc }}(t)$, which is the instantaneous phase noise of VCO. The relation between $\phi_{n, \text { inj }}(t)$ and $\phi_{n, \text { out }}(t)$ is given as

$$
\phi_{n, \text { inj }}(t)=\phi_{n, \text { out }}\left(t-\tau_{p}\right)+\phi_{m}(t)
$$

where $\tau_{p}$ is the time delay of the self-injection-locking path, and $\phi_{m}(t)$ represents a phase modulation introduced in the path due to the Doppler effect. Another relationship between $S_{\text {out }}(t)$ and $S_{\mathrm{inj}}(t)$ is their instantaneous phase difference $\alpha(t)$, which is given as

$$
\alpha(t)=\alpha_{\mathrm{inj}}+\alpha_{n}(t)
$$

where $\alpha_{\text {inj }}$ is a constant phase shift equal to $\omega_{\text {osc }} \tau_{p}$ and $\alpha_{n}(t)$ is a phase perturbation induced by $\phi_{n, \text { osc }}(t)$ and $\phi_{n, \text { inj }}(t)$. Making the Taylor's expansion of $\sin \alpha(t)$ around $\alpha_{\text {inj }}$ gives

$$
\sin \alpha(t) \approx \sin \alpha_{\mathrm{inj}}+\alpha_{n}(t) \cos \alpha_{\mathrm{inj}} .
$$

Substituting (3)-(5) and (9) into (2) leads to

$$
\begin{array}{r}
\frac{d \alpha(t)}{d t}=\frac{d \alpha_{n}(t)}{d t}=-\omega_{\mathrm{LR}} \cos \alpha_{\mathrm{inj}} \cdot \alpha_{n}(t) \\
+\frac{d \phi_{n, \mathrm{osc}}(t)}{d t}-\frac{d \phi_{n, \mathrm{inj}}(t)}{d t}
\end{array}
$$


where

$$
\omega_{\mathrm{LR}}=\frac{\omega_{\mathrm{osc}}(t)}{2 Q_{\mathrm{tank}}} \frac{E_{\mathrm{inj}}}{E_{\mathrm{osc}}} \approx \frac{\omega_{\mathrm{osc}}}{2 Q_{\mathrm{tank}}} \frac{E_{\mathrm{inj}}}{E_{\mathrm{osc}}}
$$

is interpreted as the locking range [17] of a free-running oscillator with low phase noise. Substituting (5), (6), and (10) into (1) yields the following relation between $\phi_{n, \text { out }}(t)$ and $\phi_{n, \text { osc }}(t)$ :

$$
\frac{d \phi_{n, \mathrm{out}}(t)}{d t}=-\omega_{\mathrm{LR}} \cos \alpha_{\mathrm{inj}} \cdot \alpha_{n}(t)+\frac{d \phi_{n, \mathrm{osc}}(t)}{d t} .
$$

Integrating (12) gives the instantaneous phase variation $\phi_{n, \text { out }}(t)$ with respect to the VCO output, that is

$$
\phi_{n, \text { out }}(t)=-\omega_{\mathrm{LR}} \cos \alpha_{\mathrm{inj}} \cdot \int \alpha_{n}(t) d t+\phi_{n, \mathrm{osc}}(t) .
$$

Continuing with the derivation, we take the Laplace transforms of (7), (10), and (13) with the Laplace-domain representations $\phi_{n, \text { osc }}(s), \phi_{n, \text { inj }}(s), \phi_{n, \text { out }}(s), \phi_{m}(s)$, and $\alpha_{n}(s)$ and then solve for $\phi_{n, \text { out }}(s)$ in terms of $\phi_{m}(s)$ and $\phi_{n, \text { osc }}(s)$ to yield

$$
\phi_{n, \text { out }}(s)=H_{m}(s) \cdot \phi_{m}(s)+H_{o}(s) \cdot \phi_{n, \text { osc }}(s)
$$

where, for $s \tau_{p} \ll 1$

$$
\begin{aligned}
H_{m}(s) & =\frac{\omega_{\mathrm{LR}} \cos \alpha_{\mathrm{inj}}}{s+\omega_{\mathrm{LR}} \cos \alpha_{\mathrm{inj}} \cdot\left(1-e^{-s \tau_{p}}\right)} \\
& \approx \frac{\omega_{\mathrm{LR}} \cos \alpha_{\mathrm{inj}}}{s\left(1+\omega_{\mathrm{LR}} \cos \alpha_{\mathrm{inj}} \cdot \tau_{p}\right)}
\end{aligned}
$$

shows a transfer function of integration with respect to $\phi_{m}(s)$ and

$$
\begin{aligned}
H_{o}(s) & =\frac{s}{s+\omega_{\mathrm{LR}} \cos \alpha_{\mathrm{inj}} \cdot\left(1-e^{-s \tau_{p}}\right)} \\
& \approx \frac{1}{1+\omega_{\mathrm{LR}} \cos \alpha_{\mathrm{inj}} \cdot \tau_{p}}
\end{aligned}
$$

shows a transfer function of suppression with respect to $\phi_{n, \text { osc }}(s)$. It is particularly noted that, in [18], the same expressions as (16) have been derived for a self-injection-locked oscillator. From (14)-(16), one can understand that $H_{m}(s)$ exhibits a gain that increases with decreasing modulation frequency and, thus, can greatly amplify the low-frequency modulation information of the vital signs. On the contrary, $H_{n \text {,osc }}(s)$ shows an attenuation that increases with increasing the path delay $\tau_{p}$ and thus can help suppress the phase noise if the delay time is sufficient.

To extract the phase-modulation signal, a frequency demodulator is subsequently used to process the VCO output signal. As shown in Fig. 2, the mixer multiplies $S_{\text {out }}(t)$ by its delay version $S_{\text {out }}\left(t-\tau_{d}\right)$ and outputs the signal with their phase difference to the baseband. In the time domain, this baseband signal is described by

$$
\phi_{\mathrm{BB}}(t)=\phi_{n, \text { out }}(t)-\phi_{n, \text { out }}\left(t-\tau_{d}\right)
$$

where $\tau_{d}$ is the delay time of the delay line used in the frequency demodulator. Taking the Laplace domain of (17) gives

$$
\phi_{\mathrm{BB}}(s)=\phi_{n, \mathrm{out}}(s) \cdot\left(1-e^{-s \tau_{d}}\right) .
$$

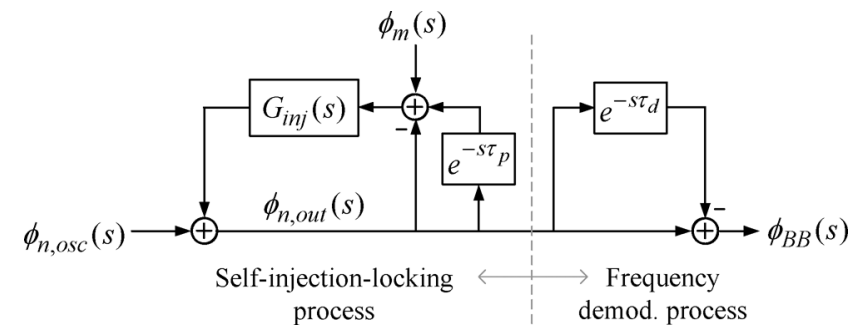

Fig. 4. Frequency-domain model of the proposed sensor architecture.

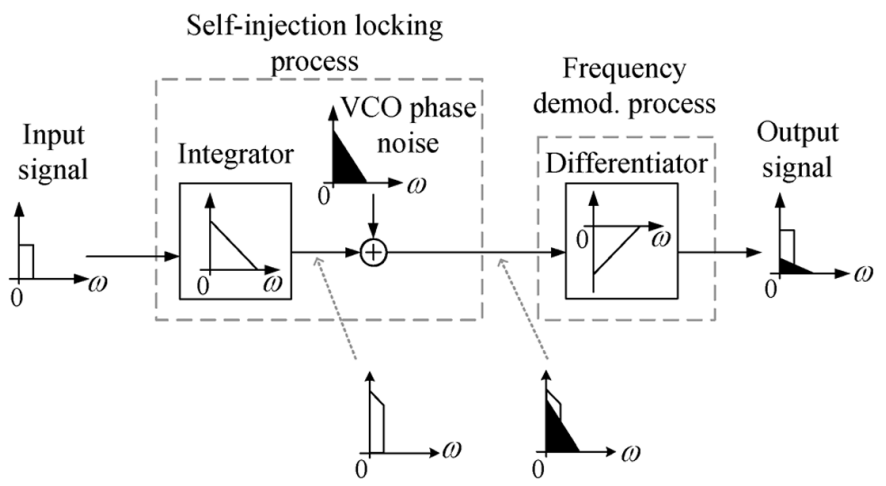

Fig. 5. Illustration of the delta-sigma noise-shaping mechanism.

For $s \tau_{d} \ll 1, \phi_{\mathrm{BB}}(s)$ in (18) is further approximated as

$$
\begin{aligned}
\phi_{\mathrm{BB}}(s) \approx & \phi_{n, \mathrm{out}}(s) \cdot s \tau_{d} \\
= & \frac{\omega_{\mathrm{LR}} \cos \alpha_{\mathrm{inj}} \cdot \tau_{d}}{1+\omega_{\mathrm{LR}} \cos \alpha_{\mathrm{inj}} \cdot \tau_{p}} \cdot \phi_{m}(s) \\
& +\frac{s \tau_{d}}{1+\omega_{\mathrm{LR}} \cos \alpha_{\mathrm{inj}} \cdot \tau_{p}} \cdot \phi_{o}(s) .
\end{aligned}
$$

According to (15), (16), and (18), the equivalent model to account for the combined process of self-injection-locking and frequency demodulation can be represented as Fig. 4 with $G_{\text {inj }}(s)=\omega_{\mathrm{LR}} \cos \alpha_{\mathrm{inj}} / s$. It is noted that this model behaves as a first-order delta-sigma modulator [19] for noise shaping to enhance the SNR of the phase-modulation signal, as illustrated in Fig. 5. The self-injection-locking mechanism acts like an integrator to reinforce the low-frequency components of the phase-modulation signal, while it functions as an attenuator to suppress the phase noise whose spectrum often has a $-30-\mathrm{dB} /$ decade slope due to the flicker noise [20]. In contrast, the frequency-demodulation process performs a differential operation to restore the phase-modulation signal without distortion and, in the meantime, push the phase noise out of the modulation bandwidth to high frequencies with a 20- $\mathrm{dB} /$ decade slope. Therefore, a phase-noise spectrum with a $-10-\mathrm{dB} /$ decade slope is predicted in the baseband. Overall, the delta-sigma process is the key for the proposed sensor system to achieve high SNR performance.

Assume that the phase-modulation signal $\phi_{m}(s)$ and the VCO phase noise $\phi_{n, \mathrm{osc}}(s)$ has a power spectral density (PSD) of $S_{m}(s)$ and $S_{n, \text { osc }}(s)$, respectively. The baseband PSD can then be represented as

$$
\begin{aligned}
S_{n, \text { out }}(s) & =\left|H_{m}(s) \cdot s \tau_{d}\right|^{2} S_{m}(s) \\
& +\left|H_{o}(s) \cdot s \tau_{d}\right|^{2} S_{n, \mathrm{osc}}(s)=S_{m}^{\prime}(s)+S_{n, \mathrm{osc}}^{\prime}(s) .
\end{aligned}
$$




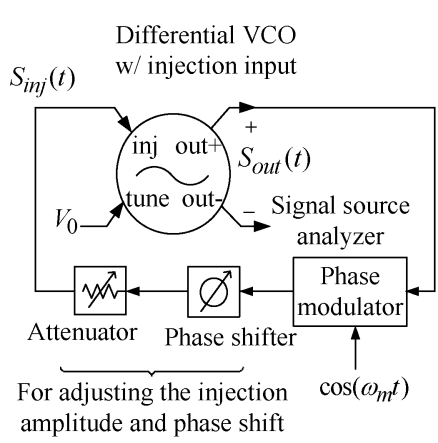

(a)

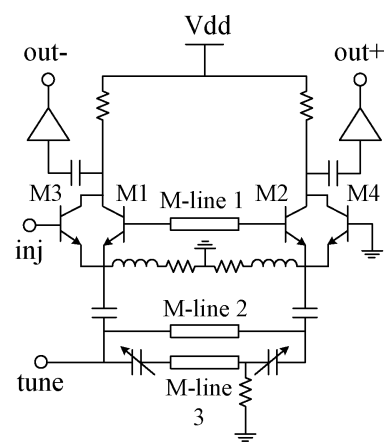

(b)
Fig. 6. SNR gain measurement. (a) Experimental setup. (b) Simplified circuit schematic of a differential VCO with an injection port.

The SNR gain is defined as the ratio between the SNR before and after the delta-sigma process, and it is given by

$$
\begin{aligned}
G_{\mathrm{SNR}}(s) & =\frac{S_{m}^{\prime}(s) / S_{n, \mathrm{osc}}^{\prime}(s)}{S_{m}(s) / S_{n, \mathrm{osc}}(s)} \\
& =\frac{\left|H_{m}(s)\right|^{2}}{\left|H_{o}(s)\right|^{2}}=\left|\frac{\omega_{\mathrm{LR}} \cos \alpha_{\mathrm{inj}}}{s}\right|^{2} .
\end{aligned}
$$

According to (21), we can predict that the SNR gain increases with decreasing modulation frequency at a rate of $20 \mathrm{~dB}$ per decade. In addition, the SNR gain increases with a larger locking range $\omega_{\mathrm{LR}}$ which, from (11), is proportional to the operating oscillation frequency $\omega_{\text {osc }}$ and the injection amplitude $E_{\text {inj }}$. We should also pay attention to the injection phase shift $\alpha_{\text {inj }}$, which causes the null detection points at every odd multiple of $90^{\circ}$. In Section III, we will conduct several experiments to verify the parametric dependence of the SNR gain as indicated above.

\section{SNR GAIN EXPERIMENTS}

Here, we provide the SNR gain measurements to verify the theory developed in Section II. Fig. 6(a) shows the experimental setup, which includes a differential VCO having an injection port, a phase modulator, a phase shifter, and an attenuator. The signal in one of the VCO's differential output terminals is phase modulated by a sinusoidal waveform of frequency $\omega_{m}$ and then fed back to the injection port of VCO via the phase shifter and the attenuator. Thus, the self-injection-locking loop is established. It is noted that both the attenuator and phase shifter are variable for adjusting the injection amplitude and phase shift. The signal in the other differential output terminal connects to a signal source analyzer for measuring the PSD. A constant voltage $V_{0}$ is applied to the VCO's tuning terminal for determining the oscillation frequency $\omega_{\text {osc }}$.

Fig. 6(b) shows a circuit schematic of the differential VCO using a push-pull configuration [21]. In the push-pull operation mode, the current circulates between the bases of the transistors M1 and M2 so as to drive the two transistors $180^{\circ}$ out of phase. Two back-to-back varactors are used in the emitter tank circuit to tune the oscillation frequency with a tuning voltage. Another differential pair of the transistors M3 and M4 forms an injection stage to receive the injection signal. Three microstrip lines are used to compensate for the circuit responses for achieving a wide tuning range. The top one helps maintain the $180^{\circ}$ phase



Fig. 7. PSD measurement results for evaluating the SNR gain.

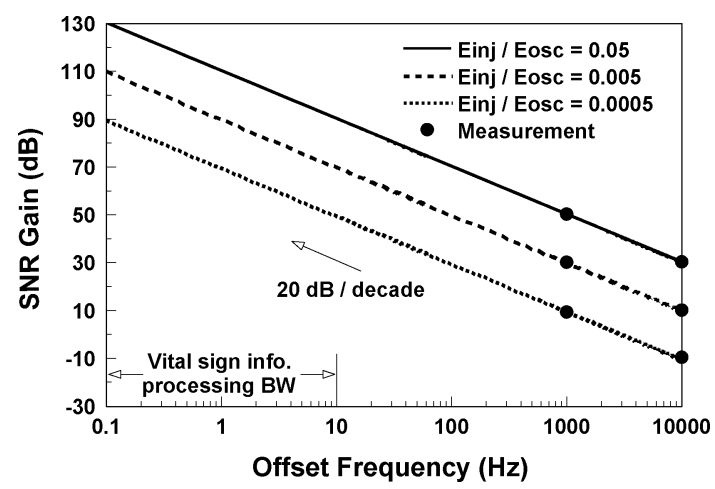

Fig. 8. SNR gain versus offset frequency with varying $E_{\mathrm{inj}} / E_{\mathrm{osc}}$.

difference between the bases of M1 and M2 at a higher operating frequency. The bottom two improve the high-frequency resonant response of the tank circuit to realize a smooth tuning over a large bandwidth. The differential output buffer stage is used to provide output isolation. For this study, two differential VCOs are implemented. One has a tuning range from 0.9 to $2.2 \mathrm{GHz}$, and the other has a tuning range from 2.2 to $3.6 \mathrm{GHz}$.

Fig. 7 plots the PSD measurement results using the experimental setup shown in Fig. 6. There are four measured curves in Fig. 7 for evaluating the SNR gain of the sensor system by means of (20) and (21). Two curves with legends of $S_{m}(s)$ and $S_{n, \text { out }}(s)$ are the measured PSDs of the injected and output phase-modulated signal, respectively, for the VCO under modulated self-injection. The other two curves with legends of $S_{n, \text { osc }}(s)$ and $S_{n, \text { osc }}^{\prime}(s)$ are the measured PSDs of the phase noise for the free-running and self-injection-locked VCO, respectively. It can be seen from Fig. 7 that the self-injection-locking notably amplifies the VCO phase-modulation signal and suppresses the VCO phase noise at the same time. With the help of the above measurement technique, SNR gain and its dependence, as expressed in (21), can be accurately verified.

Fig. 8 compares the calculated and measured SNR gains at various ratios of the injection amplitude $E_{\text {inj }}$ to the oscillation amplitude $E_{\text {inj. }}$. The solid line, broken line, and dotted line express the calculated SNR gain according to (21) with $E_{\text {inj }} / E_{\text {osc }}$ equal to $0.05,0.005$, and 0.0005 , respectively. The measured results are represented using the circle symbols, showing an excellent agreement with the calculated predictions. It is noted that, for this example, the oscillation frequency $f_{\text {osc }}$ is chosen 


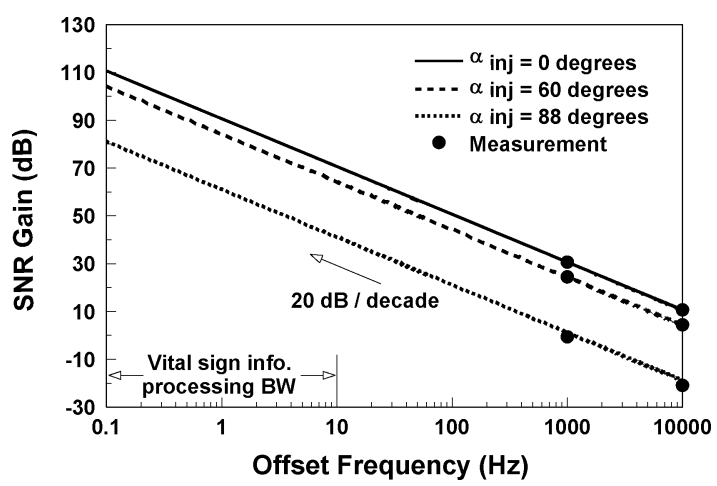

Fig. 9. SNR gain versus offset frequency with varying $\alpha_{\text {inj }}$.

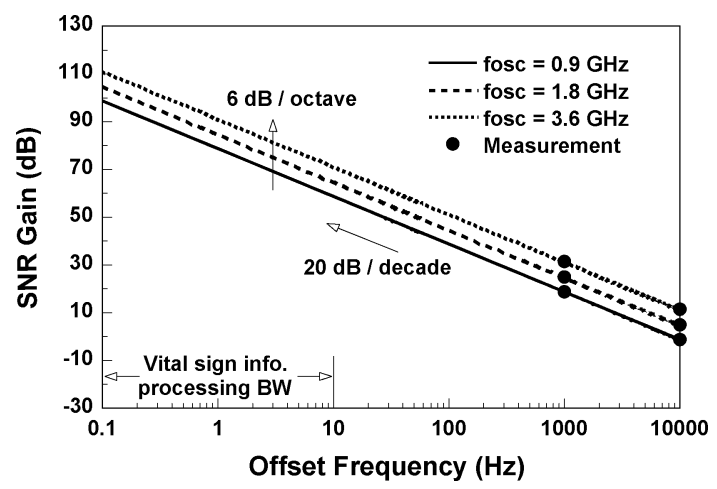

Fig. 10. SNR gain versus offset frequency with varying $f_{\text {osc }}$.

at $2.45 \mathrm{GHz}$, the tank quality factor $Q_{\text {tank }}$ is about 25 , and the injection phase shift $\alpha_{\text {inj }}$ is set at $30^{\circ}$.

With the same oscillation frequency and tank quality factor, Fig. 9 shows the comparison results for verifying the dependence of SNR gain on the injection phase shift $\alpha_{\text {inj }}$ when the ratio $E_{\text {inj }} / E_{\text {osc }}$ is set at 0.005 . The solid line, broken line, and dotted line express the calculated results with $\alpha_{\text {inj }}$ equal to $0^{\circ}$, $60^{\circ}$, and $88^{\circ}$, respectively, while the measured results are shown using circle symbols. Again, excellent agreement between calculated and measured results is obtained. We should note that, by varying $\alpha_{\text {inj }}$ only, the SNR gain achieves a maximum as $\alpha_{\text {inj }}=n \pi, n \in N$, while it vanishes as $\alpha_{\text {inj }}=(n+0.5) \pi, n \in$ $N$.

By modifying the conditions in the last example to allow a variable $f_{\text {osc }}$ and to fix $\alpha_{\text {inj }}$ at $0^{\circ}$, we can verify the dependence of SNR gain on $f_{\text {osc }}$ using Fig. 10. In a similar fashion, the calculated results are drawn with lines in Fig. 10, while the measured results are represented with symbols. The comparison again shows very good agreement. By referring to (11) and (21), the SNR gain is proportional to the square of $f_{\text {osc }}$, and it therefore exhibits an increasing rate of $6 \mathrm{~dB}$ per octave as $f_{\text {osc }}$ varies from 0.9 to $3.6 \mathrm{GHz}$.

As mentioned earlier, the SNR gain increases with decreasing modulation frequency at a rate of $20 \mathrm{~dB}$ per decade. For the vital sign sensing applications, the processing bandwidth is often limited below $10 \mathrm{~Hz}$, which corresponds to the high SNR gain region, say above $70 \mathrm{~dB}$, as shown in the solid lines of Figs. 8-10. In another aspect, the phase modulation PSD due to the Doppler effect is generally proportional to the square of the

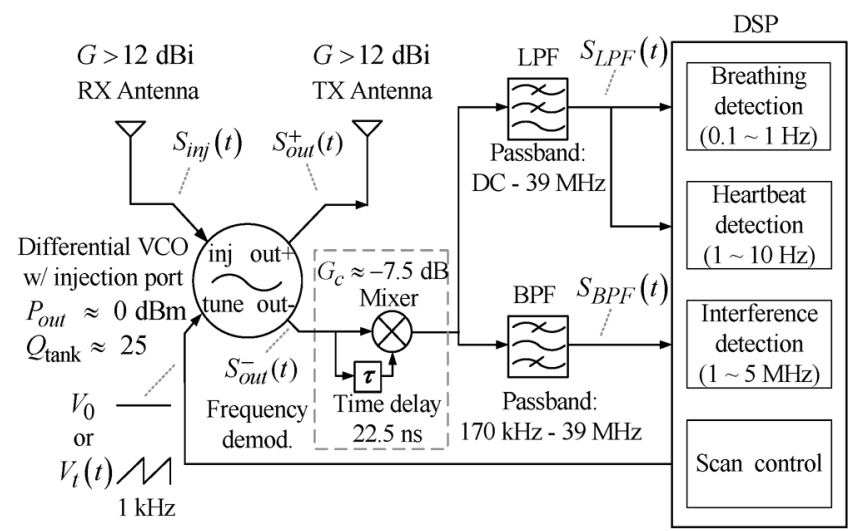

Fig. 11. Block diagram of the implemented sensor system.

operating frequency. Therefore, the inclusion of the SNR gain factor yields the SNR in proportion to the fourth power of the operating frequency. Based on this characteristic, the proposed sensor architecture can more effectively improve the sensitivity and hence the sensing distance than the conventional ones by using a higher operating frequency.

\section{VITAL-Sign SENSING EXPERIMENTS}

The block diagram of the proposed vital-sign sensor is shown in Fig. 11. The sensor system contains a receive antenna, a transmit antenna, a differential VCO with an injection port, a frequency demodulator composed of a mixer and a delay line, a low-pass filter (LPF), a bandpass filter (BPF), and a digital signal processor (DSP) with built-in analog-to-digital converter (ADC) and digital-to-analog converter (DAC). Fig. 11 also annotates the key specifications of the RF components used in this sensor system.

The sensing procedure is briefly demonstrated as follows. In transmission, the DSP delivers a frequency control word (FCW) to the built-in DAC and generates a constant voltage $V_{0}$ to select the VCO output frequency or a $1-\mathrm{kHz}$ linear ramp voltage $V_{t}(t)$ to sweep the VCO output frequency in the targeted bandwidth. The VCO output signal $S_{\text {out }}^{+}(t)$ at the differential positive terminal is then emitted toward the subject by means of the transmit antenna. In reception, the receive antenna receives the signal reflected back from the subject to serve as an injection signal $S_{\text {inj }}(t)$ into the VCO via the injection port. The received signal will include the independent wireless communication signals within the targeted bandwidth if an anti-interference ability test is carried out for the sensor system. Afterward, the VCO output signal $S_{\text {out }}^{-}(t)$ at the differential negative terminal is frequency demodulated and then low-pass or bandpass filtered for subsequently performing the vital-sign and interference detection in the DSP.

The main function of the proposed vital-sign sensor is to detect the breathing and heartbeat movements of a human subject in a noncontact way. The following shortly describes the Doppler radar principle to support this detection. Consider a distance $d$ with a time-varying displacement $x(t)$ between the sensor and the subject, and $x(t)$ results from breathing and heartbeat movements. These movements cause a Doppler shift that is proportional to the velocity of movement in the reflected signal. Therefore, the received signal's instantaneous frequency 


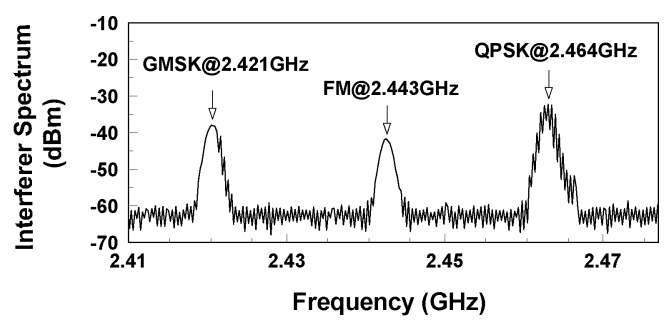

(a)

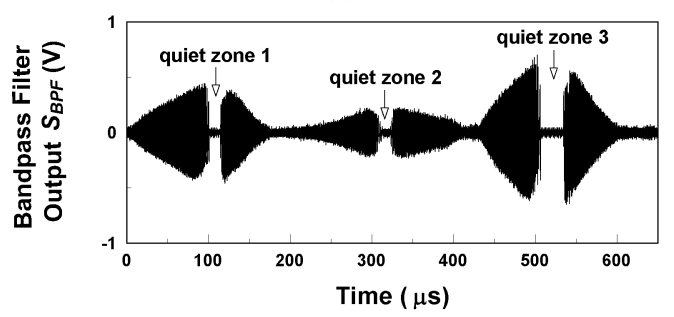

(b)

Fig. 12. Detection of radio interferences. (a) Spectrum of three interference signals including a 2.421-GHz GMSK signal, a 2.443-GHz FM signal, and a 2.464-GHz QPSK signal. (b) Baseband bandpass filter output of the sensor system in detecting the interference signals shown in (a).

is a delay version of the VCO output instantaneous frequency plus the reflected Doppler shift, that is

$$
\omega_{\text {inj }}(t)=\omega_{\text {out }}\left(t-\tau_{p}\right)+\frac{2 \omega_{\text {osc }}}{c} \cdot \frac{d x(t)}{d t}
$$

where $c$ is the speed of light and $\tau_{p}$ is the round-trip propagation delay given by $2 d / c$. Comparing (22) and (5) with substitutions of (6) and (7) yields the instantaneous phase modulation, due to the Doppler effect, given by

$$
\phi_{m}(t)=\frac{2 \omega_{\mathrm{osc}}}{c} x(t)
$$

\section{A. Sensing Results Using a Sweeping Mode}

In the swept-frequency operation mode, the VCO in the sensor system performs a frequency sweep in the industrial, scientific and medical (ISM) band from 2.4 to $2.484 \mathrm{GHz}$ at a rate of $100 \mathrm{MHz} / \mathrm{ms}$. Two experimental tests are performed on the sensor system using the swept mode. One is the anti-interference ability test, and the other is a test to eliminate the null detection points.

In the anti-interference ability test, the sensor is interfered by a combination of three independent modulation signals within the operating band. The spectrum of the combined signals has been measured by a spectrum analyzer with the same receive antenna as that of the sensor, and the results are shown in Fig. 12(a). The first one is a GMSK signal located at $f_{1}=$ $2.421 \mathrm{GHz}$ with a received power $P_{1}=-37 \mathrm{dBm}$ and $271-\mathrm{kb} / \mathrm{s}$ data rate; the second one is an FM signal located at $f_{2}=$ $2.443 \mathrm{GHz}$ with a received power $P_{2}=-42 \mathrm{dBm}$ and $320-\mathrm{kHz}$ modulation bandwidth; and the third one is a quaternary phase-shift-keying (QPSK) signal located at $f_{3}=2.464 \mathrm{GHz}$ with a received power $P_{3}=-32 \mathrm{dBm}$ and $1-\mathrm{Mb} / \mathrm{s}$ data rate. In [22], the authors have demonstrated how to extract the interference frequency and amplitude information from the

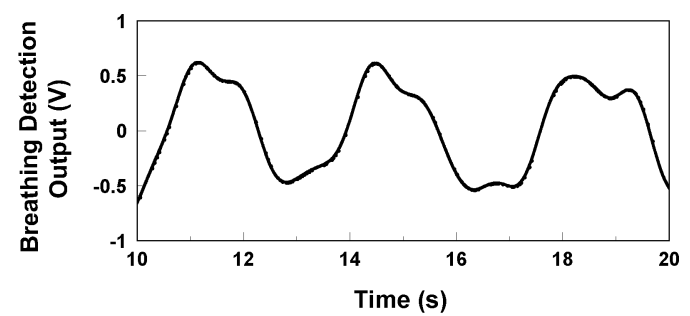

(a)



(b)

Fig. 13. Breathing detection results. (a) Output waveform. (b) Output spectrum.

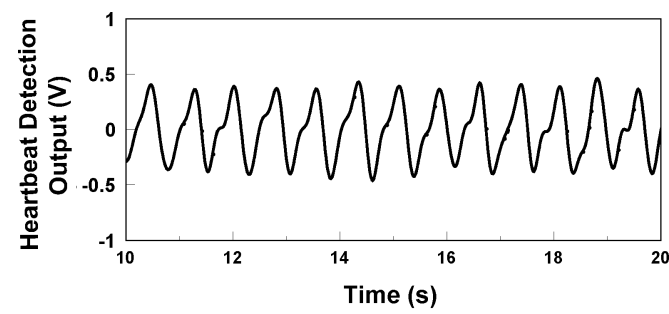

(a)

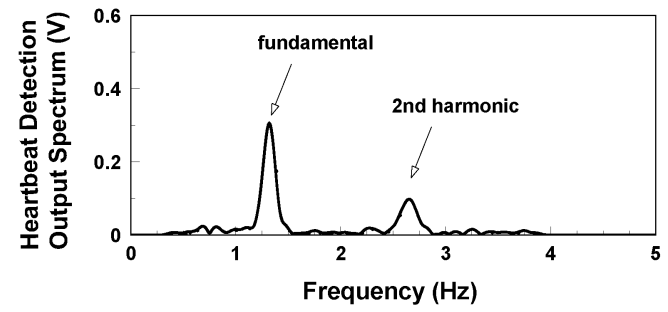

(b)

Fig. 14. Heartbeat detection results. (a) Output waveform. (b) Output spectrum.

baseband bandpass filter output signal $S_{\mathrm{BPF}}(t)$ by measuring the center position and duration of the quiet zones appearing in the response of $S_{\mathrm{BPF}}(t)$, as shown in Fig. 12(b). In this figure, three quiet zones are observed at different times, corresponding to the three interference signals at different frequencies.

In the vital-sign sensing experiment, an adult subject is seated $1 \mathrm{~m}$ from the sensor and breathing normally. Since the received signals include not only the reflected Doppler-shift signal but also the external radio interference signals in the operating band, we use an LPF to filter out the influence of the interference on the frequency-demodulated output signal and meanwhile preserve the vital sign information for DSP to process. The breathing and heartbeat information can be distinguished by applying digital filters with the following passbands: $0.1-1 \mathrm{~Hz}$ for breathing and 1-10 Hz for heartbeat. Consequently, Figs. 13(a) and (b) and 14(a) and (b) show the detected waveforms and spectrums for breathing and heartbeat, 




(a)

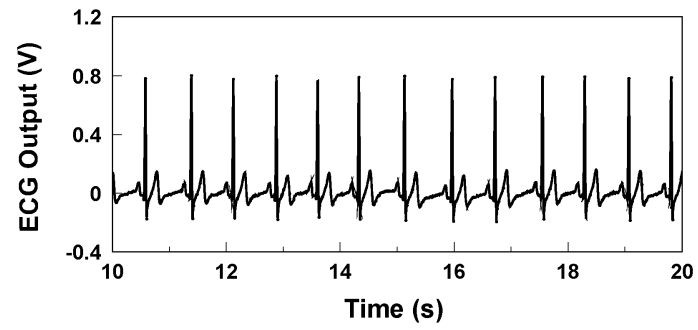

(b)

Fig. 15. Heartbeat detection results at a null detection point and verification with a simultaneous ECG recording. (a) Heartbeat detection output waveform with and without frequency sweeping. (b) ECG output waveform.

respectively. A breathing rate of 17 beats/min and a heartbeat rate of 79 beats/min can be identified.

In the performance test at the null detection point, the sensor first adjusts its operating frequency to a fixed one at 2.438 $\mathrm{GHz}$ so that the subject, seated $1 \mathrm{~m}$ away from the sensor, is at the null detection point. The heartbeat detection output for this situation is plotted by the dashed line in Fig. 15(a), showing a vanishing amplitude in the output waveform. Next, the sensor operates in the sweeping mode with the heartbeat detection output shown by the solid line in Fig. 15(a). One can see that the sweeping mode can effectively resolve the null detection point problem to provide a clear output waveform with sufficiently large amplitude.

For verifying the detected heartbeat rate, an electrocardiogram (ECG) is simultaneously recorded, as shown in Fig. 15(b), by measuring electrical potential between various points of the subject's chest using a biomedical instrumentation amplifier. The ECG provides information about heart activity and is composed of a P-wave, a QRS complex, and a T-wave to represent the depolarization and repolarization of the atria and ventricles [23]. By comparison, the two output waveforms shown by the solid lines in Fig. 15(a) and (b) coincide with each other in the same heartbeat period.

\section{B. Sensing Results at Different Operating Frequencies}

As mentioned earlier, the proposed sensor architecture can achieve an SNR in proportion to the fourth power of the operating frequency $\omega_{\text {osc }}$. This is because of the facts that both the phase-modulation PSD and SNR gain are proportional to the square of $\omega_{\mathrm{osc}}$, as can be deduced from (11), (21), and (23). Since the received power in general decreases with the square of the sensing distance, doubling the operating frequency should increase the SNR by a factor of four times. To validate this prediction, the following experiments are conducted for the sensor to detect the heartbeat at different frequencies and distances to the subject.

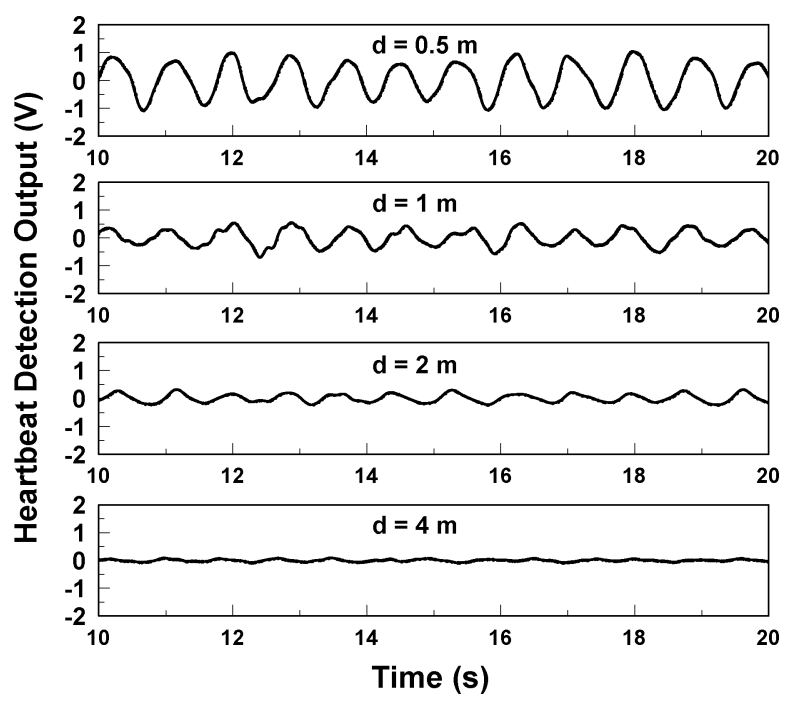

Fig. 16. Heartbeat detection results at different distances between the subject and the sensor operating at $2.4 \mathrm{GHz}$.

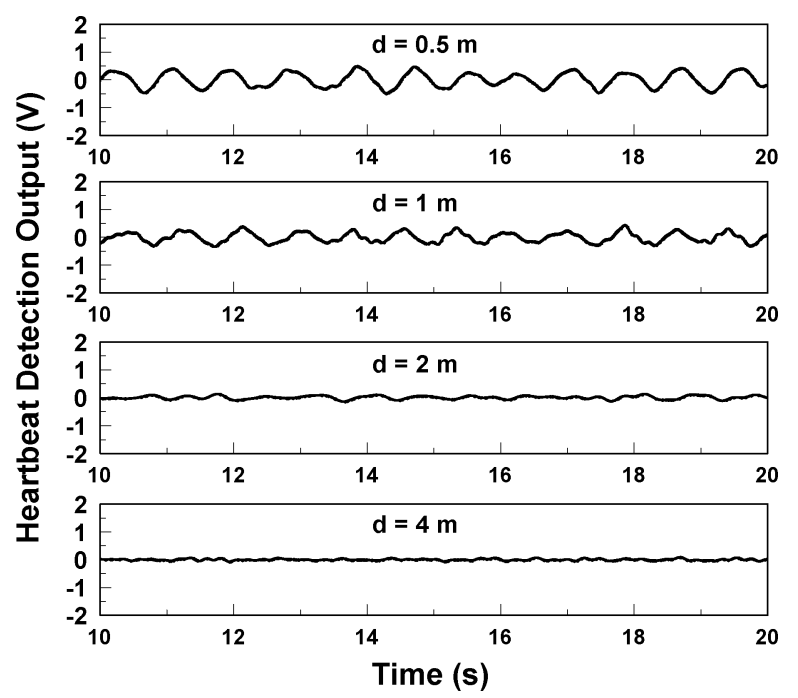

Fig. 17. Heartbeat detection results at different distances between the subject and the sensor operating at $1.8 \mathrm{GHz}$.

In the experiments, the VCO in the sensor system operates in a single-frequency mode, and its output frequencies are selected to be $1.8,2.4$, and $3.6 \mathrm{GHz}$. The sensor is placed away from the subject at four nominal distances, i.e., $d=0.5,1,2$, and $4 \mathrm{~m}$. It should be noted that the actual distances between the sensor and the subject are fine-tuned to a multiple of a half wavelength to secure the optimum SNR gain. Fig. 16 shows the heartbeat detection results at $2.4 \mathrm{GHz}$. It is observed that the amplitude of the output waveform reduces by about half as the sensing distance doubles. In particular, the digital processing of the results at $d=4 \mathrm{~m}$ fails to provide an accurate measure of the heartbeat rate, and thus the verified maximum sensing distance is $2 \mathrm{~m}$ for the sensor operating at $2.4 \mathrm{GHz}$. In a similar fashion, Figs. 17 and 18 show the heartbeat detection results at the other two frequencies, 1.8 and $3.6 \mathrm{GHz}$. Based on the same criterion of measuring the heartbeat rate, the verified maximum sensing distance is 1 and $4 \mathrm{~m}$ for the sensor operating at 1.8 and $3.6 \mathrm{GHz}$, respectively. This is consistent with our expectation 


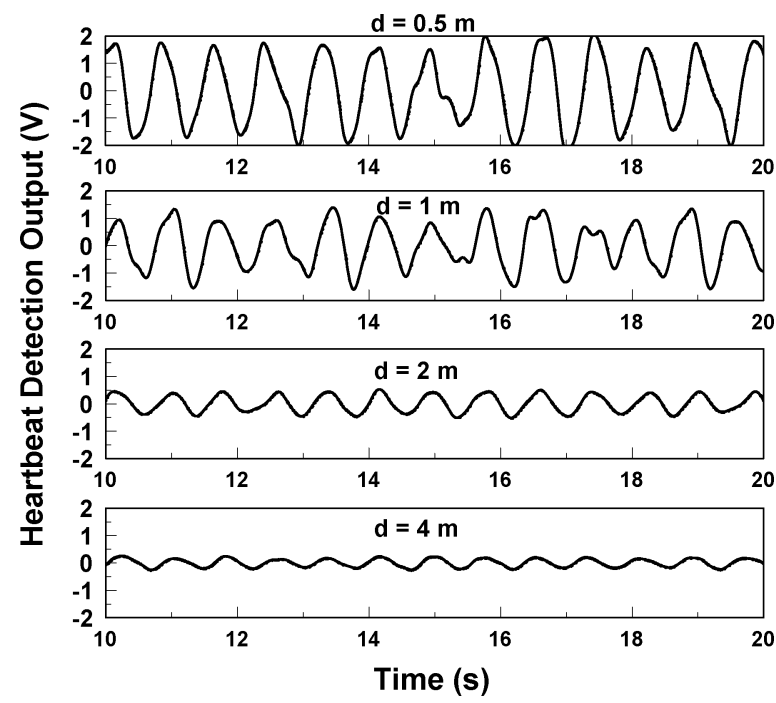

Fig. 18. Heartbeat detection results at different distances between the subject and the sensor operating at $3.6 \mathrm{GHz}$.

that the sensor can increase the sensing distance four times by doubling the operating frequency.

\section{CONCLUSION}

This paper presents a novel vital-sign sensor architecture that can resolve the interference and null detection point problems in a noncontact monitoring of cardiopulmonary motion. This paper also provides an accurate delta-sigma model to predict the parametric dependence of SNR gain for the proposed sensor architecture. The experiments verify the theoretical predictions and show very promising sensing results. The most significant results include a high-quality sensing of cardiopulmonary activities at a null detection point while interferences are introduced into the sensor system and an increase of four times the sensing distance by doubling the operating frequency.

\section{REFERENCES}

[1] C.-C. Lin, M.-J. Chiu, C.-C. Hsiao, R.-G. Lee, and Y.-S. Tsai, "Wireless health care service system for elderly with dementia," IEEE Trans. Inf. Technol. Biomed., vol. 10, no. 4, pp. 696-704, Oct. 2006.

[2] K. M. Chen, D. Misra, H. Wang, H. R. Chuang, and E. Postow, "An $X$-band microwave life-detection system," IEEE Trans. Biomed. Eng., vol. BME-33, no. 7, pp. 697-701, Jul. 1986.

[3] G. Ossberger, T. Buchegger, E. Schimback, A. Stelzer, and R. Weigel, "Non-invasive respiratory movement detection and monitoring of hidden humans using ultra-wideband pulse radar," in IEEE Ultrawideband Syst. Tech. Dig., May 2004, pp. 395-399.

[4] A. C. H. Yu, E. Cohen-Solal, B. I. Rayu, and S. Ayati, "An automated carotid pulse assessment approach using Doppler ultrasound," IEEE Trans. Biomed. Eng., vol. 55, no. 3, pp. 1072-1081, Mar. 2008.

[5] J. C. Lin, "Microwave sensing of physiological movement and volume change: A review," Bioelectromagn., vol. 13, pp. 557-565, Apr. 1992.

[6] K. M. Chen, Y. Huang, J. Shang, and A. Norman, "Microwave life-detection systems for searching human subjects under earthquake rubble or behind barrier," IEEE Trans. Biomed. Eng., vol. 27, no. 1, pp. 105-114, Jan. 2000.

[7] A. D. Droitcour, O. Boric-Lubecke, V. M. Lubecke, J. Lin, and G. T. A. Kovac, " $0.25 \mu \mathrm{m}$ CMOS and BiCMOS single chip direct conversion Doppler radars for remote sensing of vital signs," in IEEE Int. Solid State Circuits Conf. Dig., Feb. 2002, pp. 348-349.
[8] A. D. Droitcour, O. Boric-Lubecke, V. M. Lubecke, J. Lin, and G. T. A. Kovac, "Range correlation and I/Q performance benefits in single-chip silicon Doppler radars for noncontact cardiopulmonary monitoring," IEEE Trans. Microw. Theory Tech., vol. 52, no. 3, pp. 838-848, Mar. 2004.

[9] R. Fletcher and J. Han, "Low-cost differential front-end for Doppler radar vital sign monitoring," in IEEE MTT-S Int. Microw. Symp. Dig., Jun. 2009, pp. 1325-1328.

[10] J. H. Park, B. J. Jang, and J. G. Yook, "0.18 $\mu \mathrm{m}$ CMOS receiver front-end for non-invasive cardiopulmonary monitoring," in IEEE Asia-Pacific Microw. Conf. Dig., Dec. 2008, pp. 1-4.

[11] Y. Yan, C. Li, and J. Lin, "Effects of I/Q mismatch on measurement of periodic movement using a Doppler radar sensor," in Proc. IEEE Radio Wireless Symp., Jan. 2010, pp. 196-199.

[12] Y. Xiao, J. Lin, O. Boric-Lubecke, and V. M. Lubecke, "Frequency tuning technique for remote detection of heartbeat and respiration using low-power double-sideband transmission in the $K a$-band," IEEE Trans. Microw. Theory Tech., vol. 54, no. 5, pp. 2023-2032, May 2006.

[13] A. Singh and V. M. Lubecke, "A heterodyne receiver for harmonic Doppler radar cardio-pulmonary monitoring with body-worn passive RF tags," in IEEE MTT-S Int. Microw. Symp. Dig., Jun. 2010, pp. $1600-1603$.

[14] L. Chioukh, H. Boutayeb, L. Li, L. Yakia, and K. Wu, "Integrated radar systems for precision monitoring of heartbeat and respiratory status," in Asia-Pacific Microw. Conf. Dig., Dec. 2009, pp. 405-408.

[15] P. H. Lien, L. Lin, and H. R. Chuan, "Computer simulation of the RF system effects on a millimeter-wave Doppler radar for human vitalsignal estimation," in Eur. Radar Conf. Dig., Sep. 2009, pp. 465-468.

[16] F.-K. Wang, C.-J. Li, C.-H. Hsiao, T.-S. Horng, J. Lin, K.-C. Peng, J.-K. Jau, J.-Y. Li, and C.-C. Chen, "An injection-locked detector for concurrent spectrum and vital sign sensing," in IEEE MTT-S Int. Microw. Symp. Dig., May 2010, pp. 768-771.

[17] R. Adler, "A study of locking phenomena in oscillators," Proc. IRE, vol. 34, no. 6, pp. 351-357, Jun. 1946.

[18] H.-C. Chang, "Stability analysis of self-injection-locked oscillators," IEEE Trans. Microw. Theory Tech., vol. 51, no. 9, pp. 1989-1993, Sep. 2003.

[19] J. C. Candy and O. J. Benjamin, "The structure of quantization noise from sigma-delta modulation," IEEE Trans. Commun., vol. COM-29, pp. 1316-1323, Sep. 1981.

[20] T. H. Lee, The Design of CMOS Radio-Frequency Integrated Circuits, 2nd ed. Cambridge, U.K.: Cambridge Univ., 2004.

[21] A. Grebennikov, $R F$ and Microwave Transistor Oscillator Design, 1st ed. New York: Wiley, 2007.

[22] C.-J. Li, F.-K. Wang, T.-S. Horng, and K.-C. Peng, "A novel RF sensing circuit using injection locking and frequency demodulation for cognitive radio applications," IEEE Trans. Microw. Theory Tech., vol. 57, no. 12, pp. 3143-3152, Dec. 2009.

[23] J. G. Webster, Medical Instrumentation Application and Design, 4th ed. New York: Wiley, 2009.

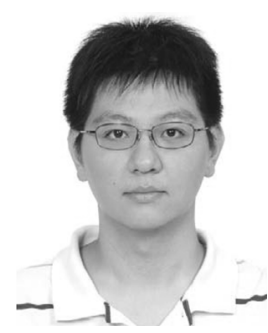

Fu-Kang Wang (S'10) was born in Kaohsiung, Taiwan, on May 15, 1985. He received the B.S.E.E. and M.S.E.E. degrees from the National Sun Yat-Sen University, Kaohsiung, Taiwan, in 2007 and 2009, respectively, where he is currently working toward the $\mathrm{Ph} . \mathrm{D}$. degree in electrical engineering.

His Ph.D. work is focused on RF sensing techniques.

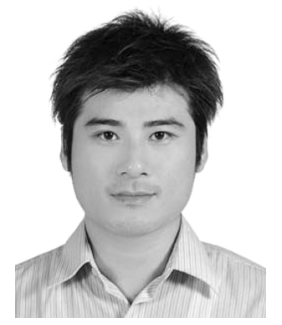

Chien-Jung Li (S'07-M'10) was born in Tainan, Taiwan, on October 26, 1979. He received the B.S.E.E. and Ph.D. degrees from the National Sun Yat-Sen University, Kaohsiung, Taiwan, in 2002 and 2009, respectively.

$\mathrm{He}$ is currently a Postdoctoral Fellow with the Department of Electrical Engineering, National Sun Yat-Sen University, Kaohsiung, Taiwan. His research interests include power-amplifier linearization techniques, frequency synthesizer designs, and local-oscillator pulling issues in direct-conversion 


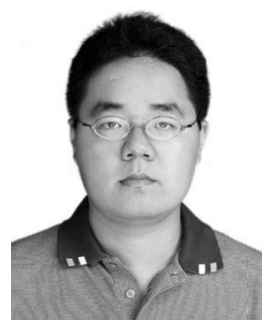

Chieh-Hsun Hsiao (S'10) was born in Kaohsiung, Taiwan, on June 5, 1984. He received the B.S.E.E. and M.S.E.E. degrees from the National Sun Yat-Sen University, Kaohsiung, Taiwan, in 2006 and 2008, respectively, where he is currently working toward the $\mathrm{Ph} . \mathrm{D}$. degree in electrical engineering.

His Ph.D. research is focused on phase- and injection-locked oscillators.

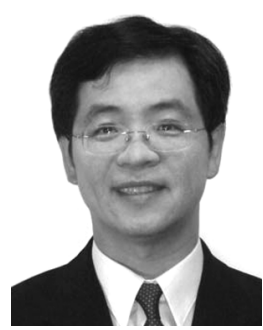

Tzyy-Sheng Horng (S'88-M'92-SM'05) was born in Taichung, Taiwan, on December 7, 1963. He received the B.S.E.E. degree from the National Taiwan University, Taipei, Taiwan, in 1985, and the M.S.E.E. and $\mathrm{Ph} . \mathrm{D}$. degrees from the University of California, Los Angeles, in 1990 and 1992, respectively.

Since August 1992, he has been with the Department of Electrical Engineering, National Sun Yat-Sen University, Kaohsiung, Taiwan, where he was the Director of the Telecommunication Research and Development Center (2003-2008) and Director of the Institute of Communications Engineering (2004-2007), and where he is currently a Professor. He has authored or coauthored over 100 technical publications published in refereed journals and conferences proceedings. He holds over ten patents. His research interests include RF and microwave integrated circuits and components, RF signal integrity for wireless system in package, and digitally assisted RF technologies.

Dr. Horng has served on several Technical Program Committees of international conferences including the International Association of Science and Technology for Development (IASTED) International Conference on Wireless and Optical Communications, the IEEE Region 10 International Technical Conference, the IEEE International Workshop on Electrical Design of Advanced Packaging and Systems, the Asia Pacific Microwave Conference, the IEEE Radio and Wireless Symposium, and the Electronic Components and Technology Conference. He was the recipient of the 1996 Young Scientist Award presented by the International Union of Radio Science, the 1998 Industry Education Cooperation Award presented by the Ministry of Education, Taiwan, and the 2010 Distinguished Electrical Engineer Award presented by the Chinese Institute of Electrical Engineering, Taiwan.

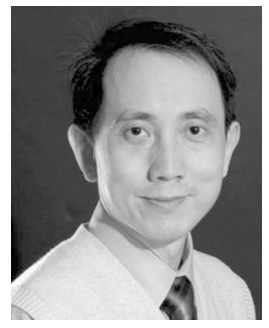

Jenshan Lin (S'91-M'94-SM'00-F'10) received the Ph.D. degree in electrical engineering from the University of California, Los Angeles, in 1994.

He was with AT\&T Bell Labs (currently Lucent Bell Labs), Murray Hill, NJ, from 1994 to 2001, and its spin-off Agere Systems from 2001 to 2003. In July 2003, he joined the University of Florida, Gainesville, as an Associate Professor and became a Professor in August 2007. He was a Visiting Professor with National Taiwan University (summer 2006) and a Visiting Researcher with NTT (summer 2010). He has authored or coauthored over 200 technical publications in refereed journals and conferences proceedings, and he holds seven patents. $\mathrm{He}$ has been serving on several conference steering committees and technical program committees, including the International Microwave Symposium (IMS), the Radio Frequency Integrated Circuits Symposium (RFIC), and the Radio and Wireless Symposium (RWS). He was the General Chair of 2008 RFIC Symposium and the Technical Program Chair of 2009 Radio and Wireless Symposium. His current research interests include sensors and biomedical applications of microwave and millimeter-wave technologies and wireless energy transmission.

Dr. Lin is an elected IEEE Microwave Theory and Techniques Society (IEEE MTT-S) Administrative Committee (AdCom) member, currently serving as the Chair of Technical Coordinating Committee. He served as an Associate Editor for the IEEE TRANSACTIONS ON MICROWAVE THEORY AND TECHNIQUES from 2006 to 2010. He was the recipient of the 1994 UCLA Outstanding Ph.D. Award, the 1997 Eta Kappa Nu Outstanding Young Electrical Engineer Honorable Mention Award, and the 2007 IEEE MTT-S N. Walter Cox Award.

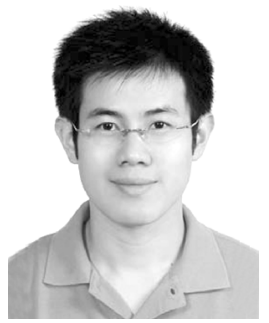

Kang-Chun Peng (S'00-M'05) was born in Taipei, Taiwan, on February 18, 1976. He received the B.S.E.E., M.S.E.E., and Ph.D. degrees from the National Sun Yat-Sen University, Kaohsiung, Taiwan, in 1998, 2000, and 2005, respectively.

$\mathrm{He}$ is currently an Assistant Professor with the Department of Computer and Communication Engineering, National Kaohsiung First University of Science and Technology, Kaohsiung, Taiwan. His current research interests are in the area of delta-sigma modulation techniques, low-noise phase-locked loops, low-power voltage-controlled oscillators, and modulated frequency synthesizers.

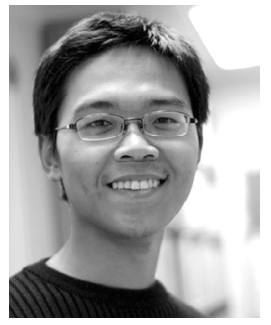

Je-Kuan Jau was born in Tainan, Taiwan, on November 19, 1977. He received the B.S.E.E. and $\mathrm{Ph}$.D. degrees from National Sun Yat-Sen University, Kaohsiung, Taiwan, in 1999 and 2006, respectively.

$\mathrm{He}$ is currently with the Information and Communications Research Laboratories, ITRI, Hsinchu, Taiwan, as an Engineer. His research interests are RF transmitters and power amplifiers.

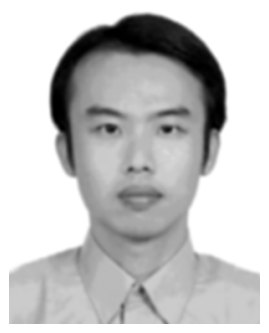

Jian-Yu Li (S'07) was born in Pingtung, Taiwan. He received the B.S.E.E and M.S.E.E degrees from the National Sun Yat-Sen University, Kaohsiung, Taiwan, in 1998 and 2000, respectively. He is currently working toward the Ph.D. degree in communication engineering at National Chiao Tung University, Hsinchu, Taiwan.

In 2000, he joined the Information and Communications Research Laboratories, ITRI, Hsinchu, Taiwan, as an Engineer. His research interests are in the area of microwave integrated circuits and passive

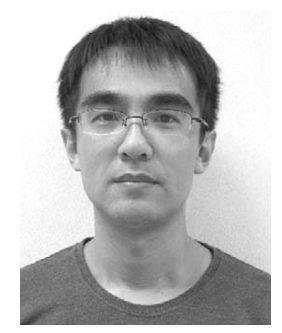

Cheng-Chung Chen was born in 1975 in Hsinchu, Taiwan. He received the B.S. degree in electrical engineering from the National Sun Yet-Sen University, Kaoshiung, Taiwan, in 1997, and the M.S. and Ph.D. degrees in communication engineering from the National Chiao-Tung University, Hsinchu, Taiwan, in 1999 and 2003, respectively.

In 2003, He joined the Information and Communications Research Laboratory, ITRI, Hsinchu, Taiwan. His research interests include RF front-end chip and module design. 